\title{
SENTIDO DE COHERENCIA Y FOBIA SOCIAL: UN ESTUDIO DESCRIPTIVO
}

\author{
Ana Isabel Burguillos Peña \\ Doctora en Psicología. Psicóloga Interna Residente \\ (Hospital Juan Ramón Jiménez, Huelva) \\ anaisabel.burguillos@gmail.com \\ Manuel Acosta Contreras \\ Catedrático EU Psicología de la \\ Educación (Universidad de Huelva) \\ acosta@uhu.es
}

Fecha de Recepción: 24 Febrero 2019

Fecha de Admisión: 30 Abril 2019

\section{RESUMEN}

Antecedentes. El sentido de coherencia es una variable que engloba las siguientes dimensiones: Comprensibilidad (es posible comprender lo que sucede); Manejabilidad (recursos necesarios para solucionar los problemas están a disposición de la persona); y Significatividad (experiencia de que los retos se merecen los esfuerzos). Las personas con un alto SOC muestran menor presencia de sintomatología psicosomática, de síntomas depresivos (Flannery y Flannery, 1990), menores indicadores de psicopatología y menores problemas de salud mental (Terja, et al., 2006). Objetivos. El estudio se dirigió a conocer de modo descriptivo la variable "sentido de coherencia" en 20 personas con diagnóstico de fobia social de una USMC. Método. El diseño de la investigación fue de tipo descriptivo en el que se pretendían conocer las puntuaciones de la variable "sentido de coherencia" evaluado a través del cuestionario SOC-13 de Antonovsky, en una muestra de 20 personas con niveles leves de depresión (medido mediante el BDI-II de Beck) y diagnóstico de fobia social. Se midió a través del FNE el temor a la evaluación negativa. Resultados. Las personas del estudio muestran niveles bajos en Sentido de Coherencia, siendo el rango de puntuaciones posibles de 13 a 91 puntos, obtuvieron una media de 19,78. Conclusiones. Los resultados confirman las conclusiones arrojadas por los diferentes estudios previamente analizados, pudiendo observar cómo se encuentran puntuaciones inferiores de "sentido de coherencia" en personas con síntomas psicopatológicos (Flannery y Flannery, 1990), y más concretamente en ansiedad social (Burguillos, 2014). Hubo una relación negativa entre el SOC y el temor a la evaluación negativa. Por ello, se hace necesario poner en práctica herramientas psicoterapéuticas basadas en este constructo salutogénico como medio de proteger la salud mental.

Palabras claves: sentido de coherencia; salutogénesis; ansiedad Social 


\section{ABSTRACT}

Sense of coherence and social fobia: a descriptive study. Antecedents. The sense of coherence is a variable that encompasses the following dimensions: Comprehensibility (it is possible to understand what happens); Manageability (resources needed to solve problems are available to the person); and Significance (experience that the challenges deserve the efforts). People with a high SOC show a lower presence of psychosomatic symptomatology, depressive symptoms (Flannery \& Flannery, 1990), lower indicators of psychopathology and fewer mental health problems (Terja, et al., 2006). Objective. The study was aimed at knowing in a descriptive way the variable "sense of coherence" in 20 people diagnosed with social phobia of a USMC. Method. The design of the research was of a descriptive type in which it was intended to know the scores of the variable "sense of coherence" evaluated through the SOC-13 questionnaire of Antonovsky, in a sample of 20 people with mild levels of depression (measured by Beck's BDI-II) and diagnosis of social phobia._The fear of negative evaluation was measured through the FNE Results. The people in the study show low levels in the sense of coherence, being the range of possible scores of 13 to 91 points, they obtained an average of 19.78. Conclusions. The results confirm the conclusions of the different studies previously analyzed, being able to observe how lower scores of "sense of coherence" are found in people with psychopathological symptoms (Flannery and Flannery, 1990), and more specifically in social anxiety (Burguillos, 2014). There was a negative relationship between the SOC and the fear of negative evaluation.Therefore, it is necessary to implement psychotherapeutic tools based on this salutogenic construct as a means of protecting mental health.

Keywords: sense of coherence; salutogenesis; social anxiety

\section{INTRODUCCIÓN}

Aaron Antonovsky, médico y sociólogo nacido en 1923 en los Estados Unidos comenzó en los años 70 a desarrollar la perspectiva salutogénica. En Antonovsky creció un importante interés en la investigación sobre las clases sociales y la salud, siendo más tarde objeto de su interés, el impacto del estrés en la salud. Cuando la Salud Pública se centró en los factores de riesgo y la enfermedad, surgió la atención sobre el estrés como un evento negativo que incrementaría la susceptibilidad y el riesgo de la gente a enfermar. Para Antonovsky, el estrés forma parte de la vida de las personas (Lindström y Eriksson, 2006).

La aproximación salutogénica se centraba por tanto en los recursos para la salud y los procesos de promoción de la salud. No se trataría por tanto, de un rasgo específico del individuo ni un estilo de afrontamiento, sino de un conjunto de competencias cognitivas que proporcionan una orientación disposicional generalizada (Moreno- Jiménez, González y Garrosa, 2001). Antonovsky se pregunta por qué se mantiene la salud, en lugar de por qué enfermamos, por lo que introduce en su modelo el concepto de Sentido de Coherencia, al que se hará referencia a partir de aquí como SOC, y los Recursos Generales de Resistencia o RRGs (Eriksson y Lindström, 2008).

Los conceptos principales que Antonovsky desarrolla con esta nueva teoría son los Recursos Generales de Resistencia (RGRs) y el Sentido de Coherencia. Los Recursos Generales de Resistencia son aquellas características de una persona, grupo o contexto que facilitan una gestión eficaz de las demandas internas o externas que se consideran estresores. Se trataría por tanto, de recursos personales, interpersonales o contextuales de los que pueden disponer los individuos en caso de necesitarlos. Se refieren a factores biológicos, materiales y psicosociales que facilitan a las personas la percepción de su vida como coherente, estructurada y comprensible. Los RGRs más usuales son el dinero, el conocimiento, la experiencia, la autoestima, los hábitos saludables, el compromiso, el apoyo social, la cultura, la inteligencia, las tradiciones y la visión de la vida. Son todos 
aquellos medios que facilitan el empleo de conductas adaptativas, saludables y como respuesta adaptativa frente a estresores (Fernández, 2009).

Si una persona tiene todos estos recursos, le será muy fácil enfrentarse a los desafíos y contratiempos que le surjan en la vida, pero con eso no basta, según Antonovsky, es necesaria la capacidad para utilizar estos recursos, a la que llamó Sentido de Coherencia (SOC. Sense of Coherence), concepto clave de su teoría, para el que diseñó su propio cuestionario para medirlo. El Sentido de Coherencia (SOC) de Antonovsky se encuentra dentro de su modelo salutogénico, y tiene como objetivo principal medir una orientación global de la personalidad, la cual, facilita la solución de problemas de una forma adaptativa ante situaciones estresantes. Es la capacidad de la persona de percibir el significado del mundo que le rodea, así como adquirir la relación entre las acciones y las consecuencias de éstas en el entorno. Así, pretendía Antonovsky explicar por qué algunas personas permanecían saludables en situaciones estresantes, mientras que otras enfermaban. De este modo, concebía el sentido de coherencia como una tendencia disposicional relativamente estable, que nos hace evaluar las circunstancias como significativas, predecibles y manejables. Antonovsky diferencia en este constructo tres componentes: comprensibilidad (componente cognitivo), manejabilidad (componente instrumental o de comportamiento) y significatividad (componente motivacional), (Rivera de los Santos et al., 2011).

El primero, comprensibilidad, se refiere al grado en el que las personas tienen un sentido cognitivo de los estímulos con los que se encuentran en el presente y en el futuro. Se considera el componente cognitivo del constructo, y se refiere a la habilidad de establecer conexiones lógicas y ordenadas en lo que sucede en el ambiente y en creer que la vida es predecible. El segundo, manejabilidad, hace referencia al grado en el que los individuos entienden que los recursos adecuados para hacer frente a las demandas del entorno se encuentran a su disposición. Es el componente instrumental o conductual del constructo. Se relaciona con la autoeficacia y la competencia, pero a diferencia de ésta, no considera el control de los recursos. El último componente es el de significatividad, que es el valor otorgado por la persona a aquello que sucede, se refiere al deseo de la persona, a los valores, a las emociones, al sentimiento de que la vida merece la pena. Una persona con un alto nivel de significatividad cree que merece la pena invertir su energía en todos aquellos retos y demandas que le rodean, por ello, este componente tiene un carácter más emocional y es considerado como el aspecto motivacional del constructo (Antonovsky, 1994).

La ansiedad es una respuesta innata cuyo fin es la supervivencia. Cuando nos encontramos ante un peligro la ansiedad se dispara haciendo que reaccionemos de la forma más eficaz para sobrevivir, por lo tanto, la ansiedad es beneficiosa, ya que nos ayuda a enfrentarnos a situaciones difíciles. Pero también puede ocurrir que la ansiedad nos traicione, como en situaciones que no suponen ningún riesgo, pero que la persona evalúa como amenazantes (subir un ascensor) 0 cuando ante una situación concreta, necesita actuar de manera especial (un examen). Ante estas explicaciones se puede afirmar que los existen problemas de ansiedad cuando se sufre ante situaciones en las que la mayoría de las personas no se ponen ansiosas o cuando se experimenta en un grado extremo en situaciones donde la mayoría de la gente se sentiría sólo moderadamente ansiosa (Pastor y Sevilla, 2005).

La fobia social es un miedo irracional y desproporcionado a un cierto número de situaciones de interacción social. La persona está totalmente convencida de que su actuación va a ser inadecuada, ridícula, embarazosa o humillante. Esa convicción se acompaña de sensaciones físicas como taquicardia, sudoración temblores, etc. Para afrontar las situaciones en las que se siente mal la persona las afronta evitándolas 0 si se encuentra en ellas pone en marcha una serie de maniobras con el fin de que los demás no se den cuenta de lo que le sucede. Entre las situaciones sociales que más 


\section{SENTIDO DE COHERENCIA Y FOBIA SOCIAL: UN ESTUDIO DESCRIPTIVO}

malestar provocan, están: asistir a reuniones sociales, hablar en público, pedir información, relacionarse con figuras de autoridad, conocer gente nuevas, etc. Se pueden diferenciar dos subtipos de fobia social:

Fobia social específica: Ios miedos aparecen centrados en una gama mínima de situaciones sociales. La más corriente es la de hablar en público, ya que a excepción de que la persona esté acostumbrada a ello, genera cierta incomodidad. Sin embargo, podemos hablar de fobia social cuando el malestar es muy intenso o cuando la conducta social, hablar, no se ejecuta o se entorpece marcadamente por el alto nivel de ansiedad.

Fobia social generalizada: se da cuando los temores aparecen en una amplia gama de situaciones sociales. En algunos casos las situaciones temidas incluirían todas las situaciones sociales menos aquellas en las que el fóbico se relaciona con personas "seguras" que a menudo son familiares muy directos 0 amigos muy íntimos.

La fobia social, al igual que otros tipos de trastornos, se compone de tres elementos (Rivero y Pastor, 2012):

Cognitivo: hace referencia a los pensamientos catastróficos, que aparecen en primer lugar sobre la propia actuación, que ya se anticipa que será inadecuada. A continuación la persona piensa que los demás se van a dar cuenta y por tanto le van a juzgar en términos muy negativos. Finalmente, piensan que ese juicio despectivo se convertirá en un rechazo personal.

Fisiológico: los cambios que se producen porque nuestro sistema nervioso autónomo se prepara para afrontar una situación difícil. Cuando percibimos una situación como peligrosa, en nuestro cerebro se segregan ciertas sustancias que envían señales al SNA que dispara todos los signos de ansiedad: aceleración cardiaca, aumento del ritmo respiratorio, dilatación pupilar, entre muchos otros. Estos cambios que se producen son adaptativos, tienen una misión.

Motor: en el caso de la fobia social se refiere a la ejecución voluntaria de comportamientos destinados a no sentir ansiedad, a que externamente no se note (evitación o escape).

El sentido de coherencia se entiende como un factor promotor de la salud, puesto que es la variable que favorece el uso de los recursos adecuados conducentes a conductas saludables. A través del sentido de coherencia, entendemos las situaciones estresantes como comprensibles, manejables mediante los recursos de los que disponemos, y significativas para nuestra existencia personal. Las personas con un SOC fuerte, se verán capaces de afrontar situaciones estresantes empleando estrategias centradas en resolver el problema del modo más adaptativamente posible. Sin embargo, aquellas personas que poseen un SOC más débil, serán menos eficaces a la hora de afrontar determinadas situaciones estresantes (citado en Burguillos, 2015).

El sentido de coherencia, además de haber sido estudiado y relacionado con la salud física y mental, encontrando asociaciones inversas entre el SOC y ansiedad, depresión, quejas psicosomáticas, o patologías cardíacas o cáncer, entre otras; también se ha observado que mantiene una relación, en este caso positiva con variables emocionales. El SOC se ha visto relacionado negativamente con estados emocionales negativos, y de forma positiva, con aquellos estados emocionales favorables para la salud (Moksnes, Espnes, y Lillefjell, 2012). El estado emocional, y en concreto, los síntomas depresivos y la ansiedad de los adolescentes, se han visto influidos por el sentido de coherencia. Éste es una variable predictora significativa de los estados emocionales, siendo esta relación inversa (Blom et al, 2010).

El sentido de coherencia, en un estudio sobre la percepción de la salud y la satisfacción laboral en docentes universitarios, a través de su componente de "comprensibilidad" disminuiría la probabilidad de sufrir disfunción social (87.2\%) y la "significatividad" actuaría como protectora de los síntomas depresivos (96.7\%) (Burguillos, 2015). El sentido de coherencia también se relaciona negativamente con la ansiedad social en estudiantes universitarios (Burguillos, 2014). 


\section{OBJETIVOS}

El estudio se dirigió a conocer de modo descriptivo la variable "sentido de coherencia" en 20 personas con diagnóstico de fobia social de una USMC.

\section{MUESTRA}

Los participantes de la muestra fueron escogidos por criterio de accesibilidad, no probabilística. Eran un total de 20 pacientes (15 mujeres y 5 hombres) de una Unidad de Salud Mental Comunitaria (USMC), todos con diagnóstico inicial de Fobia Social (CIE- 10 F40.1). Del total de la muestra, 15 (75\%) son mujeres, y $5(25 \%)$ son hombres. La media de edad de los sujetos es de 25,32 años. En cuanto al estado civil, se pueden identificar 18 (90\%) personas "solteras" (5 hombres y 13 mujeres); el resto (20\%) convive con sus parejas. Del total de personas, el $90 \%$ se encuentran trabajando.

\section{MÉTODO}

El diseño de la investigación fue de tipo descriptivo en el que se pretendían conocer las puntuaciones de la variable "sentido de coherencia" en una muestra de 20 personas con niveles leves de depresión comórbidos y diagnóstico de fobia social presenta en la historia clínica inicial.

\section{Instrumentos}

\section{Sentido de Coherencia}

El constructo de sentido de coherencia se mide con el Cuestionario de Orientación hacia la Vida (OLQ) de Antonovsky (1985). El Cuestionario SOC-13 (Antonovsky, 1993) consta de 13 ítems que se responden en una escala tipo Likert con siete puntuaciones. El rango de puntuaciones posibles oscila entre 13 y 91 . Se incluyen tres escalas correspondientes a las tres dimensiones del constructo: comprensibilidad, manejabilidad y significatividad.

\section{Síntomas Depresivos}

Se ha empleado el Inventario de Depresión de Beck BDI-II para evaluar la sintomatología depresiva en esta muestra. Se trata de un autoinforme compuesto por 21 ítems de tipo Likert. Es un cuestionario que recoge la presencia y gravedad de los síntomas y reflejan los criterios de los manuales diagnósticos DSM-IV-TR y CIE-10. Las puntuaciones oscilan entre 0 y 63, siendo los puntos de corte: 0-13, mínima depresión; 14-19, depresión leve; 20-28, depresión moderada; y 29-63, depresión grave.

\section{Ansiedad Social}

La Escala de Miedo a la Evaluación Negativa (Fear of Negative Evaluation Scale, FNES) (Watson y Friend, 1969) mide la intensidad del miedo que experimentan los sujetos ante la evaluación negativa por parte de los demás. Consta de 30 ítems y se usa como una medida de los síntomas cognitivos, respondiendo a los modelos cognitivos de la ansiedad y fobia social. Este instrumento ha demostrado su validez empírica tanto en pacientes con fobia social como en sujetos normales (Musa, Kostogianni y Lepine, 2004). Tiene un rango de puntuaciones de 0 a 30 con ítems dicotómicos. Una puntuación superior a 15 en la escala total nos indicaría que la persona tiene fobia social, siendo evidentemente ésta una medida de grado. 


\section{RESULTADOS}

\section{Descriptivos Variables}

\section{Sentido de Coherencia}

La puntuación total del SOC presenta una media de 19,78. Las dimensiones del "sentido de coherencia" son significatividad, manejabilidad y comprensibilidad. Se han obtenido unas medias de $21,48,17,97$, y 19,89 respectivamente. La FNES muestra puntuaciones medias de 22,3. Se dividieron en dos grupos: niveles bajos $(<15)$ y altos $(>15)$ de "temor a evaluación negativa". El nivel más bajo obtuvo una media de 14,8 y el grupo de mayor puntuación mostró 29,8 puntos en la escala FNES.

\section{Relación entre Sentido de Coherencia y Fobia Social}

Las personas con mayor puntuación en esta escala obtuvieron una menor puntuación en el SOC $(14,53)$, mientras que el grupo más bajo (con menos síntomas de fobia social) se relacionaron con una mayor puntuación en "sentido de coherencia" $(25,03)$. Se podría llegar a estudiar este dato en profundidad como medida predictiva de remisión parcial o total en el diagnóstico de fobia social.

\section{DISCUSIÓN}

Los sujetos con mayor sintomatología de Fobia Social presentan una media inferior en "sentido de coherencia", mientras que los que muestran menores síntomas autoinformados a pesar del diagnóstico de Fobia Social tienen una media superior. Los resultados confirman las conclusiones arrojadas por los diferentes estudios previamente analizados (Burguillos, 2014), pudiendo observar cómo se encuentran puntuaciones inferiores de "sentido de coherencia" en personas con síntomas psicopatológicos (Flannery y Flannery, 1990), y más concretamente en ansiedad social (Burguillos, 2015). Hubo una relación negativa entre el SOC y el temor a la evaluación negativa. Por ello, se hace necesario poner en práctica herramientas psicoterapéuticas basadas en este constructo salutogénico como medio de proteger la salud mental.

En la literatura existen pocos estudios que analicen la relación del sentido de coherencia y la fobia social, pero sí se ha estudiado el efecto mediador frente a la ansiedad o depresión, como una forma de afrontamiento para reducir el malestar psicológico (Antonovski y Sagy, 2001; 1991; Frenz, Carey y Jorgensen, 1993; Fernández, 2009).

\section{CONCLUSIONES}

Relación entre el Sentido de Coherencia y la Ansiedad Social. Se puede observar que la media de los casos clínicos (>15 puntos en FNES) de Fobia Social (FS) es ligeramente inferior $(14,53)$ al grupo de "no casos" (<15), con una media de 25,03 . Habría que realizar estudios correlacionales y predictivos para llegar a una conclusión firme en esta relación, puesto que se encuentran limitaciones en cuanto al establecimiento de relaciones causales en los estudios de tipo descriptivo.

\section{REFERENCIAS BIBLIOGRÁFICAS}

Acosta, M. y Burguillos-Peña, A.I. (2014). Estrés y Burnout en profesores de primaria y secundaria de Huelva: las estrategias de afrontamiento como factor de protección. International Journal of Developmental and Educational Psychology, 4 (1), 303-310. ISSN: 0214-9877.

Antonovsky, A. (1994). Antonovsky's sense of coherence concept: an instrument for primary prevention in social work services. International Social Work, 511-522.

Antonovsky, H. y Sagy, S. (2001). The development of a sense of coherence and its impact on res- 
ponses to stress situations. The Journal of Social Psychology, 126(2), 213-225.

Blom, E.C.H., Serlachius, E., Larsson, J.O., Theorell, T. e Ingvar, M. (2010). Low sense of coherence (SOC) is a mirror of general anxiety and persistent depressive symptoms in adolescents girls -across-sectional study of a clinical and a non- clinical cohort. Health and Quality of Life Outcomes, 8, 1-13.

Burguillos, Al. (2014). Sentido de coherencia e inteligencia emocional: efecto en la ansiedad social de estudiantes universitarios. International Journal of Developmental and Educational Psychology, 4 (1), 295-302. ISSN: 0214-9877

Burguillos, Al. (2015). La inteligencia emocional y el sentido de coherencia en la percepción de la salud de los docentes universitarios. Tesis Doctoral. Recuperado de: https://dialnet.unirioja.es/servlet/tesis?codigo $=46891$

Caballo, V., Salazar, I.C., García-López, L.J., Irurtia, M.J. (2011). Fobia Social: características clínicas y diagnósticas. p.172-206. En Caballo, V., Salazar, I.C. y Carrobles, J.A. (2011) Coords. Manual de Psicopatología y Trastornos Psicológicos. Madrid: Pirámide.

Eriksson, M. y Lindstrom, B. (2008). A salutogenic interpretation on the Ottawa Charter. Health Promotion International, 23, 190-198.

Fernández, M. E. (2009). Estrés percibido, estrategias de afrontamiento y sentido de coherencia en estudiantes de enfermería: su asociación con salud psicológica y estabilidad emocional (Tesis Doctoral no publicada). Universidad de León, León.

Flannery, R.B. y Flannery, G.J. (1990). Sense of coherence, life stress and psychological distress: a prospective methodological inquiry. Journal of Clinical Psychology, 46, 415-420.

Frenz, W., Carey, P., y Jorgensen, S. (1993). Psychometric evaluation of Antonovsky's Sense of Coherence Scale. Psychological Assessment, 5(2), 145-153. Doi: http://dx.doi.org/10.1037/1040-3590.5.2.145

Inglés, C.J.; Méndez, F.X.; Hidalgo, M.D.; Rosa, A.I.; y Orgilés, M. (2003). Cuestionarios, Inventarios y Escalas de Ansiedad Social para Adolescentes: una revisión crítica. Revista de Psicopatología y Psicología Clínica, 8(1), 1-21.

Lindstrom, B. y Eriksson, M. (2006). Contextualizing salutogenesis and Antonovsky in public health development. Health Promotion International, 21, 238-244. Recuperado de: http://heapro.oxfordjournals.org/content/21/3/238.full.pdf+html.

Moksnes, U.K., Espnes, G.A. y Lillefjell, M. (2012). Sense of coherence and emotional health in adolescents. Journal of Adolescence, 35(2), 433-441.

Moreno-Jiménez, B., González, J. y Garrosa, E. (2001). Desgaste profesional (burnout), personalidad y salud. En J. Buendía y F. Ramos (Eds.) Empleo, Estrés y Salud, pp. 59-83. Madrid: Pirámide.

Moreno, B., Alonso, M. y Álvarez, E. (1998). Sentido de Coherencia, Personalidad Resistente, Autoestima y Salud. Revista de Psicologia de la Salud, 13(2), 185- 207.

Musa, C. Kostogianni, N. y Lepine, J. P. (2004). The Fear of Negative Evaluative Scale (FNE): psychometric properties of the French version. Encephale, 30, 517-524.

Palacios-Espinosa, X., Restrepo-Espinosa, M.H. (2008). Aspectos conceptuales e históricos del sentido de coherencia propuesto por Antonovsky: ¿una alternativa para abordar el tema de la salud mental?. Informes Psicologicos, 10(11), 275- 300.

Pastor, A., Zamora, R., y Rivero, R. (eds.) (2012). Intervención Práctica en Habilidades Sociales y de la Comunicación. Murcia: Servicio de Publicaciones de la AEPCCC.

Pastor, C., Sevilla, J., (2005). "Tratamiento psicológico de la fobia social".

Publicaciones del Centro de Terapia de Conducta. 
Pastor, C., Sevilla, J., (2005). "Tratamiento psicológico del pánico-agorafobia".

Publicaciones del Centro de Terapia de Conducta.

Rivera, F., Ramos, P., Moreno, C. y Hernán, M. (2011). Análisis del Modelo Salutogénico en España: aplicación en Salud Pública e implicaciones para el Modelo de Activos en Salud. Revista Española de Salud Pública, 85(1), 137- 147.

Rivero, R. y Pastor, A. (eds.) (2012). Cómo intervenir paso a paso y sesión por sesión en Trastornos de Ansiedad. Murcia: Servicio de Publicaciones de la AEPCCC.

Watson, D. y Friend, R. (1969). Measurement of social evaluative anxiety. Journal of Consulting and Clinical Psychology, 33, 448-457. 